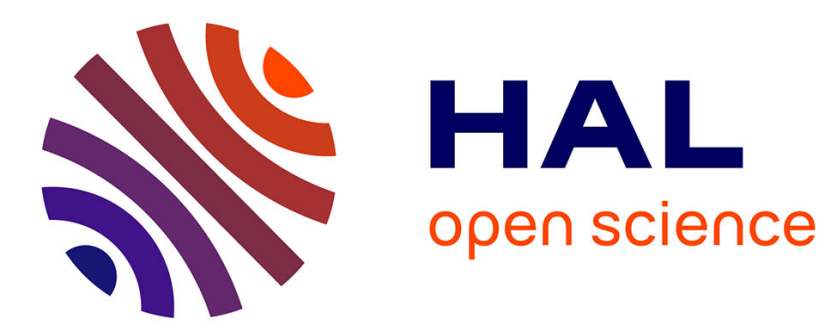

\title{
Electron plasma density deduced from lower oblique resonance measurements
}

\author{
J. Thiel, L.R.O. Storey, J.P. Lebreton
}

\section{To cite this version:}

J. Thiel, L.R.O. Storey, J.P. Lebreton. Electron plasma density deduced from lower oblique resonance measurements. Physics of Fluids, 1984, 27 (2), pp.506. 10.1063/1.864614 . insu-02886048

\section{HAL Id: insu-02886048}

\section{https://hal-insu.archives-ouvertes.fr/insu-02886048}

Submitted on 31 Aug 2020

HAL is a multi-disciplinary open access archive for the deposit and dissemination of scientific research documents, whether they are published or not. The documents may come from teaching and research institutions in France or abroad, or from public or private research centers.
L'archive ouverte pluridisciplinaire HAL, est destinée au dépôt et à la diffusion de documents scientifiques de niveau recherche, publiés ou non, émanant des établissements d'enseignement et de recherche français ou étrangers, des laboratoires publics ou privés. 


\title{
Electron plasma density deduced from lower oblique resonance measurements
}

\author{
J. Thiela) and L. R. O. Storey ${ }^{\text {b) }}$ \\ Laboratoire de Physique et Chimie de 1'Environnement, Centre National de la Recherche Scientifique, 45045 \\ Orléans Cédex, France \\ J. P. Lebreton \\ European Space Research and Technology Centre, Space Plasma Physics Division, Noordwijk ann Zee, \\ Netherlands
}

(Received 11 May 1982; accepted 31 October 1983)

\begin{abstract}
In a magnetoplasma, the amplitude of the lower oblique resonance in the mutual impedance of two electric antennas is very sensitive to the plasma frequency. This property can be used to measure the electron density in a simple way when the electron temperature and the magnetic field are known, provided also that the probe has been properly calibrated. As an application, densities are deduced from measurements made by a mutual-impedance probe on a rocket in the auroral ionosphere. In the future, better practical design and theoretical modeling of the probe should make calibration unnecessary.
\end{abstract}

\section{INTRODUCTION}

In a magnetoplasma, the potential created by a point source of alternating current exhibits a resonance in the frequency range of propagation of the whistler mode, the upper limit of which is either the electron gyrofrequency or the plasma frequency, whichever is the smaller. The amplitude and the frequency of this so-called "lower oblique resonance" depend on the electron density and temperature, on the static magnetic field strength and on the vector distance from the source to the point of observation; they depend not only on the length of this vector, but also on its direction relative to the field. These dependences can be observed by means of a mutual-impedance probe consisting of two electric antennas, one excited by a transmitter so as to emit a sinusoidal current $I$, and the other connected to a receiver which measures the induced potential difference $V$. The mutual impedance of the probe, $Z=V / I$, is related in a simple way to the potential of a point source, provided the elements of the antennas are sufficiently small and they are not perturbed by the structure that supports them.

Up to now, the diagnostic use of observations of the lower oblique resonance (LOR) made by means of mutualimpedance probes has been limited to deducing various plasma parameters from the direction or frequency of the resonance, or from those of the related oscillatory structure. The electron density $n_{e}$ or temperature $T_{e}$ have been measured in the laboratory ${ }^{1-3}$ and in space, ${ }^{4,5}$ by comparing the experimental data on these directions or frequencies with their theoretical values: in a similar way, the field-aligned drift velocity $V_{\|}$of the thermal electrons has been measured in the laboratory, ${ }^{3}$ though not yet in space. When the plasma frequency is substantially larger than the electron gyrofre-

\footnotetext{
a) Present address: Groupe de Recherches sur l'Energétique des Milieux Ionisés, Université d'Orléans, 45045 Orléans Cédex, France.

b) Present address: Space, Telecommunications, and Radio Science Laboratory, Stanford Electronics Laboratories, Stanford, California 94305.
}

quency, which is often the case in the ionosphere, these properties of the resonance are insensitive to variations of $n_{e}$, so this parameter is measured inaccurately.

In the present paper, a method is proposed for deducing the electron density from the amplitude of the lower oblique resonance. This method is applied to the interpretation of data from the medium-frequency probe aboard the Porcupine $F 3$ rocket launched from Kiruna, Sweden $\left(21^{\circ} \mathrm{E}, 68^{\circ} \mathrm{N}\right)$ on 19 March 1979, at 22.57 UT.

\section{INSTRUMENT}

As sketched in Fig. 1, the probe sensor consisted of four similar units numbered from 1 to 4 , each comprising a spherical electrode $3 \mathrm{~cm}$ in diameter connected to some miniature electronics housed in a small metal box at a distance $d \simeq 10 \mathrm{~cm}$. The line joining the centers of two adjacent electrodes had a length $r=93.2 \mathrm{~cm}$ and made an angle $\beta=32.5^{\circ}$ with the rocket axis, which was nominally aligned with the magnetic field. Only two adjacent units were used at a time, for the duration of a single frequency sweep $(0.137 \mathrm{sec})$, dur-

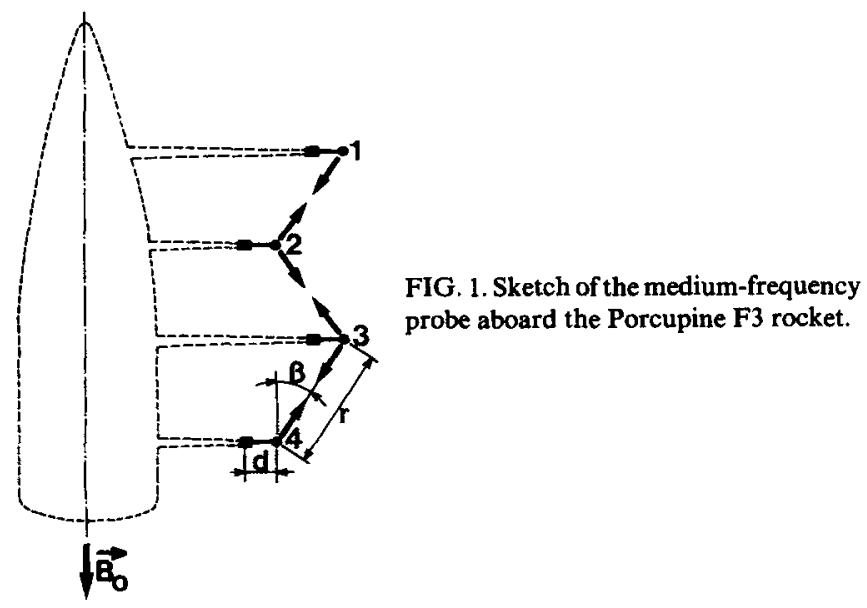


ing which each unit either emitted a radio-frequency (rf) current or received an if potential alternately, so as to make reciprocal measurements of the mutual impedance function. The frequency range covered was $0.1-1.5 \mathrm{MHz}$, and different pairs of adjacent units were activated on successive sweeps. The complete instrument, comprising the sensor together with the transmitter and receiver, will be referred to here as the medium-frequency ( $\mathrm{mf}$ ) probe.

Before the experiment, we considered that each unit of the sensor would behave as a monopole antenna, while the body of the rocket payload would serve both as a ground electrode for the return of the emitted current and as a reference point for the measurement of the received potential. On this view, the mutual impedance could be calculated theoretically by modeling the sensor as a pair of monopoles. Moreover, we assumed that the spherical electrodes of the emitting and receiving monopoles could be modeled, respectively, as a point current source and as a point contact with the plasma.

\section{RESULTS}

Figure 2 presents four typical curves of the received signal strength versus frequency. The curve (i) was recorded at the beginning of the flight, when the sensor was still stowed in the payload and the active electrodes were connected together through a calibration capacitance of $561 \mathrm{pF}$. The curves (ii) and (iii) were recorded at altitudes (and times after launch) of $462.4 \mathrm{~km}(387.6 \mathrm{sec})$ and $385.4 \mathrm{~km}(503.4$ $\mathrm{sec}$ ), respectively. The lower oblique resonance peak, which is lower and wider in (iii) than in (ii), occurs around $0.7 \mathrm{MHz}$ in both cases; its width is governed by $n_{e}$ and $T_{e}$ jointly. The weak secondary peak around $0.2 \mathrm{MHz}$, which corresponds to the peak in the calibration curve (i), is of technical origin. In addition, curve (ii) exhibits a minimum at the electron gyrofrequency of $1.3 \mathrm{MHz}$, which was expected theoretically; above it, the signal strength increases as the frequency rises towards the plasma and upper-hybrid resonance fre-

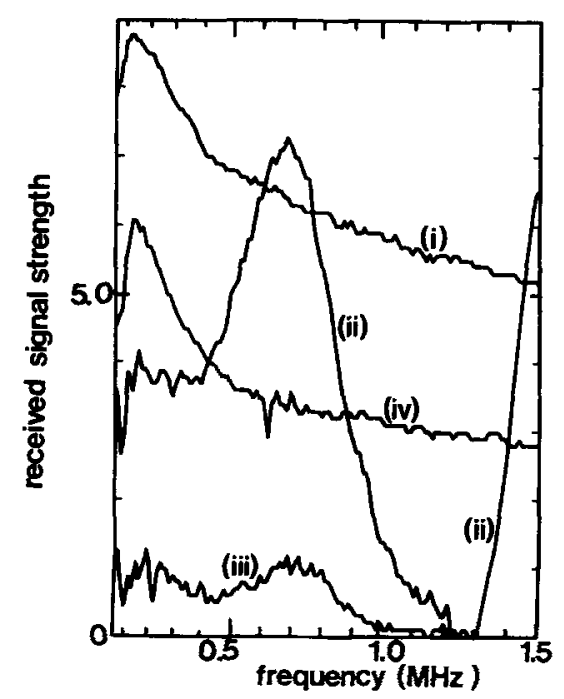

FIG. 2. Typical experimental curves recorded during the flight. The vertical scale is linear, with arbitrary units. quencies. The curve (iv) shows the data from the last frequency sweep, recorded at $79.1 \mathrm{~km}(665.1 \mathrm{sec})$ near the end of the flight.

After smoothing and averaging, the complete set of calibration curves such as (i) was used in the analysis of the response curves measured with the sensor deployed, such as curves (ii)-(iv). At any given frequency, the ratio of the measured signal strength to the strength of the calibration signal gave the ratio of the modulus of the mutual impedance of the sensor to the modulus of the impedance of the calibration capacitance. In this way, the modulus of the mutual-impedance function was determined without having to know either the emitted current or the gain of the receiver.

\section{INTERPRETATION}

Adopting the model outlined at the end of Sec. II, the mutual-impedance function of the sensor could be calculated theoretically for any given pair of values of the electron density $n_{e}$ and temperature $T_{e}$, with the help of a program that computes the scalar potential created by an alternating point charge in a Maxwellian magnetoplasma. ${ }^{6}$ This program uses the least-damped-root approximation, which involves solving the dispersion equation $\epsilon\left(\omega, k_{\|}, k_{1}\right)=0$ for electrostatic waves, selecting the root $k_{\perp}\left(k_{\|}, \omega\right)$ with the smallest imaginary part, and using only this root in the calculation of the potential ${ }^{7}$; here $\omega$ is the angular frequency, $k_{\|}$ and $k_{\perp}$ are the components of the wavenumber vector $\mathbf{k}$ parallel and perpendicular to the magnetic field, and $\epsilon$ is the longitudinal dielectric function. Calculations made in this way, with the given values of the angle $\beta$ and of the distance $r$ between the electrodes, yield the results presented in Fig. 3. In this figure, three normalized parameters are used: $\boldsymbol{\Omega}_{p}$ is the plasma frequency $f_{p}$ normalized with respect to the cyclotron frequency $f_{c}$ of the electrons, $R$ is the distance $r$ normalized to the electron Larmor radius $r_{L}$ and $\zeta_{0}$ is the magnitude of the mutual impedance at the lower oblique resonance normalized to its vacuum value. Since $\Omega_{p}$ is proportional to $n_{e}^{1 / 2}$ and $R$ to $T_{e}^{-1 / 2}$, the chart in Fig. 3 defines an $\left(n_{e}, T_{e}\right)$ plane; in it, the labeled lines correspond to constant values of $\xi_{0}$. It shows that, so long as the plasma frequency is comparable with or larger than the electron gyrofrequency, the amplitude of the lower oblique resonance in the mutual impedance depends more strongly on $n_{e}$ than on $T_{e}$; the dependence on $n_{e}$ is approximately as $n_{e}^{-1}$. Hence, the mutual impedance of a pair of point monopole antennas is very sensitive to the electron density.

When $f_{c}$ and $T_{e}$ are known, the electron density $n_{e}$ and the related error $\Delta n_{e}$ can be deduced graphically or numerically from a measured value of $\zeta_{0}$ with the help of the chart. For instance, at the time when the curve (ii) in Fig. 2 was recorded, other instruments aboard the rocket measured $1220 \pm 10 \mathrm{kHz}$ for $f_{c}$ and $1150 \pm 100 \mathrm{~K}$ for $T_{e}$, whence $R$ lies in the range 51.4-57.2, with 54.1 as the most probable value. On the other hand, since the theoretical value of the mutual impedance between two monopoles separated by $93.2 \mathrm{~cm}$ in a vacuum corresponds to $104 \mathrm{pF}$, a direct comparison between curves (i) and (ii) gives $\zeta_{0}=0.221 \pm 0.005$. The procedure represented by the lower hatched area in Fig. 3 leads to $2.73<\Omega_{p}<2.90$ (most probably 2.83 ), whence 


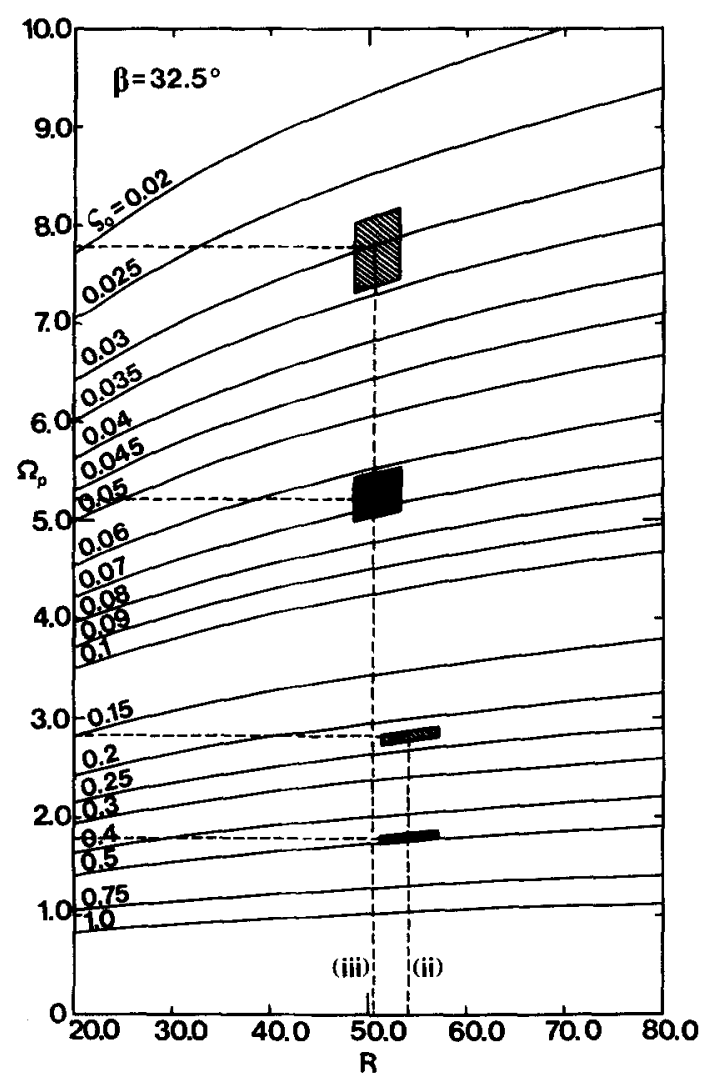

FIG. 3 Iso- $\zeta_{0}$ curves in the $\left(R, \Omega_{p}\right)$ plane for $\beta=32.5^{\circ}$.

$3303 \mathrm{kHz}<f_{p}<3567 \mathrm{kHz}$ and $n_{e}$ in the range 1.35 $1.58 \times 10^{11} \mathrm{~m}^{-3}$, with $1.48 \times 10^{11} \mathrm{~m}^{-3}$ as the most probable value. In the same way, the measured values of $f_{c}, T_{e}$, and $\xi_{0}$ are, respectively, $1260 \pm 10 \mathrm{kHz}, 1400 \pm 100 \mathrm{~K}$, and $0.031 \pm 0.003$ for the curve (iii), whence $n_{e}=1.19 \times 10^{12}$ $\mathrm{m}^{-3}$ (upper hatched area in Fig. 3). This analysis was applied to the 1672 frequency sweeps of the mf probe during the Porcupine F3 flight, using the electron temperatures from the retarding-potential analyser of Spenner and Ott (private communication).

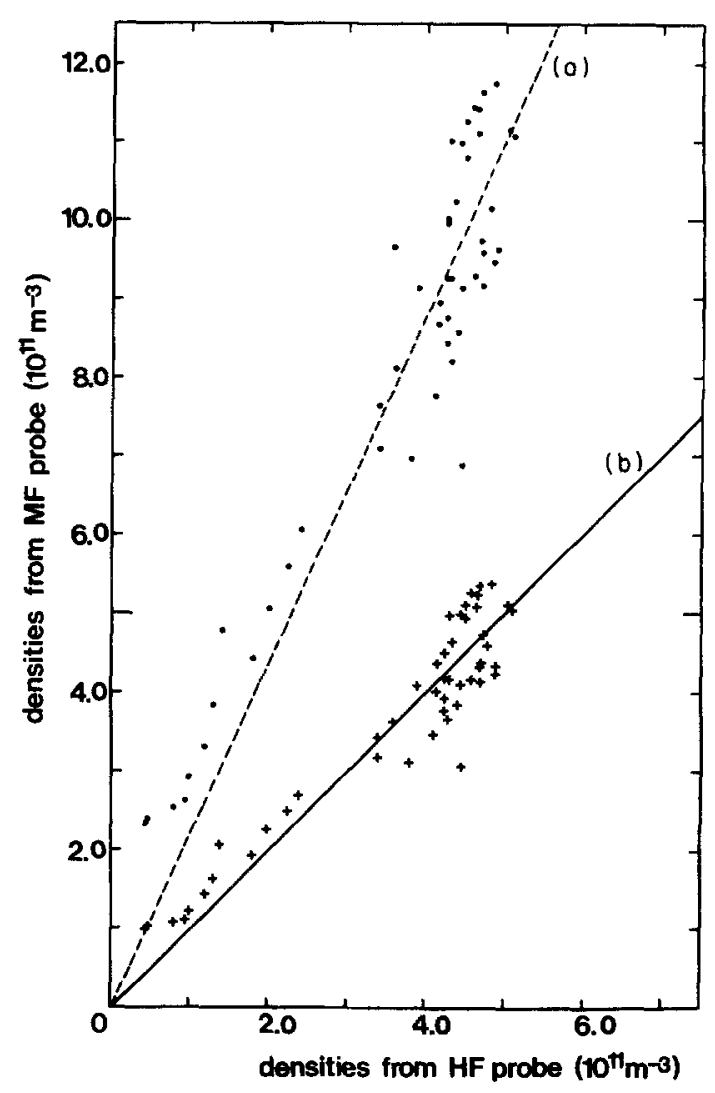

FIG. 4. Regression diagram of the electron density given by the mf probe versus that given by the hf probe: (a) using the amplitudes of the lower oblique resonance deduced directly from the $\mathrm{mf}$ probe measurements; and (b) after multiplying the above amplitudes by the factor 2.18 .

In Fig. 4, the densities obtained in this way are graphed, as dots, against those obtained from a high-frequency $(0.8$ 14.5 MHz) quadripole mutual-impedance probe on the same payload; the latter are believed to be accurate to a few percent, except when the hf probe is perturbed by the payload wake. Both sets of measurements have been averaged over 10 sec periods. Apart from some scattering, the two measure-

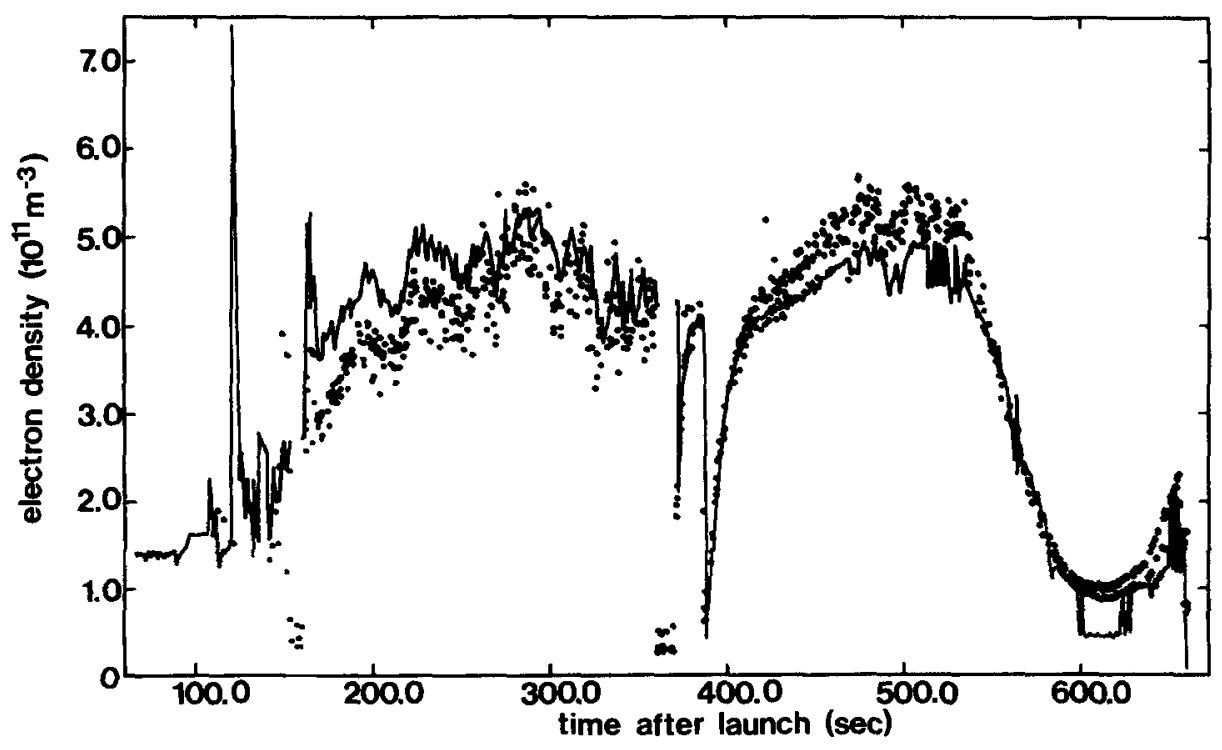

FIG. 5. Electron density obtained from the high-frequency (-) and the medium-frequency (...) probes, versus time after launch. 
ments of $n_{e}$ are proportional to one another as they should be; their regression line is shown dashed in the figure. However, the density given by the $\mathrm{mf}$ probe is higher, by a factor of about 2.20, than that given by the hf probe. Another statement of this result is that the two determinations of $n_{e}$ would agree if the measurements of $|\boldsymbol{Z}|$ for the $\mathrm{mf}$ probe were multiplied by 2.18, as is represented by the crosses in Fig. 4 .

By applying this correction to the data shown in Fig. 2, we obtained $5.4 \times 10^{10} \mathrm{~m}^{-3}<n_{e}<6.4 \times 10^{10} \mathrm{~m}^{-3}$ (most probably $5.85 \times 10^{10} \mathrm{~m}^{-3}$ ) from the curve (ii) and $4.8 \times 10^{11} \mathrm{~m}^{-3}<n_{e}<6.2 \times 10^{11} \mathrm{~m}^{-3} \quad$ (most probably $5.35 \times 10^{11} \mathrm{~m}^{-3}$ ) from the curve (iii). Accordingly, the hatched areas should be replaced by the black areas in Fig. 3 .

We have duly made this empirical correction to the data from the $\mathrm{mf}$ probe, and the values of $n_{e}$ obtained from both probes have been graphed versus time after launch in Fig. 5. Although the corrected densities are slightly lower than those given by the hf probe before rocket apogee, and higher afterwards, the mf probe yields useful results so long as it has been calibrated properly, in this instance by the hf probe. The slight differences are not yet understood but an interpretation of the large disagreement between the present experimental behavior of the $\mathrm{mf}$ probe and the theory, i.e., a ratio of the measured to the theoretical mutual impedance as large

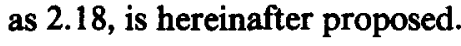

\section{DISCUSSION}

Some clues to the origin of the discrepancy are to be found in Fig. 2. A comparison between the calibration curve (i) and the experimental curve (iv) shows that, when the latter was recorded, the rocket had left the ionosphere and was effectively in a vacuum. This is inferred from the proportionality of the two signals, which have the constant ratio 1.81 above $0.4 \mathrm{MHz}$; below this frequency the ratio varies slightly, due perhaps to the presence of a residual plasma of very low density. The part of the record (iv) above $0.4 \mathrm{MHz}$ can be used to calculate the mutual impedance between two adjacent sensor units, which is capacitive; it corresponds to a capacitance of $561 \times 1.81=1015 \mathrm{pF}$. This measurement contrasts with the theoretical value of $104 \mathrm{pF}$ for two monopoles separated by $93.2 \mathrm{~cm}$; the ratio of the measured to the theoretical capacitance is 9.76 . This discrepancy, a factor of almost 10 , is in the same sense but larger than the one already noted in the analysis of the ionospheric measurements. Since it occurs in free space, plasma phenomena cannot be responsible, so its origin should be relatively easy to discover.

Part of it is due to stray capacitance in the electronics of the sensor units. In-flight measurements of the vacuum input capacitance of the emitting sensor unit yielded $4 \pm 1 \mathrm{pF}$, to which the self-capacitance of the spherical emitting electrode contributed only $1.67 \mathrm{pF}$; thus the strays amounted to $2.3 \pm 1 \mathrm{pF}$, an unexpectedly high value. In a vacuum, therefore, only the fraction $1.67 / 4=0.42$ of the nominal radiofrequency current was actually emitted. Stray capacitance in the receiving sensor unit attenuated the received rf potential by the same fraction. Altogether, considering the experimental errors in the measurement of the input capacitance, the combined effects of stray capacitance in the two sensor units divided the received signal strength, and hence the mu- tual impedance, by a factor in the range 3.2-9.0, with 5.7 as the most probable value; this left a factor of 1.1-3.0 (most probably 1.7) to be explained in some other way.

The likeliest explanation of this remaining discrepancy is that it is due to perturbation of each of the two spherical electrodes by the supporting structure, in particular by the box that houses the electronics. Assuming that a charge $q$ on the emitting electrode induces a charge $-\alpha q$ on its associated electronics box, and that an equivalent perturbation affects the receiving electrode, a reduction of the mutual impedance by the factors just cited requires $\alpha$ values in the range $0.05-0.42$ (most probably 0.23 ). Rough theoretical estimates of $\alpha$ were made electrostatically, replacing the box, which had the form of a parallelipiped, by a sphere, the diameter of which was varied between limits equal to the length and the width of the box. The results indicated that $\alpha$ lay between 0.2 and 0.3 , so the explanation seems plausible.

The question thus arises as to whether the same two influences on the sensor electrodes, namely stray capacitance and electrostatic perturbation, can explain the discrepancy between the theoretical and experimental values of the mutual impedance when the sensor is inside the ionosphere; this, it will be recalled, amounted to a factor of 2.18 .

In the plasma, the self-impedance of the emitting sensor unit around the LOR frequency appeared to be mainly capacitive, and equivalent to $12 \pm 2 \mathrm{pF}$. Assuming $2.3 \mathrm{pF}$ for the strays, the apparent capacitance of the spherical electrode in the plasma was $9.7 \mathrm{pF}$, which again is unexpectedly high. Taken at its face value, it implies that the combined effects of the stray capacitances in the emitting and receiving sensor units should reduce the mutual impedance by the factor $(12 /$ $9.7)^{2}=1.53$, the limits of error being 1.43 and 1.69 .

Thus, of the observed factor 2.18 , only a factor 1.29 1.52 (most probably 1.42 ) remains to be explained in terms of perturbation of the electrodes by the supporting structure. With the same model as was used for the sensor in a vacuum, but with the influence of the plasma on the mutual impedance taken into account, values of the parameter $\alpha$ in the range $0.16-0.24$ are required to explain this factor. The most probable value is 0.20 , which is almost the same as was found for the vacuum; this would be expected if the plasma could be treated as an isotropic dielectric medium. While we have not been able to solve the electrostatic problem of estimating $\alpha$ for the sensor unit in an anisotropic plasma, and so we have no solid theoretical grounds for thinking that it should be the same as in a vacuum, we consider that the experimental coincidence lends some support to the model.

Summarizing, the discrepancy between the experimental and theoretical values of the mutual impedance at the LOR appears to be explicable as the joint effects of stray capacitance in the sensor units, and of perturbation of the electrodes by the supporting structure. There is no need as yet to invoke another possibility, which is that the modeling of the electrodes as points might be unsatisfactory. This conclusion should be treated with due reserve, since neither the experimental measurements of self-impedance nor the theoretical calculations of the electrostatic perturbation were precise.

If this conclusion is accepted, however, it follows that in 
any future experiments with a mf mutual-impedance probe, the discrepancy could be eliminated by proper design of the sensor units. This is a matter both of curtailing the causes, and of allowing more accurately for their residual effects. First, the electronics should be redesigned so as to reduce the stray capacitance to the strict minimum. Second, the measurement of the self-impedance should be made more accurate, so that the effects of the residual stray capacitance can be allowed for. Finally, the perturbation due to the supporting structure should be reduced by increasing the distance between the spherical electrode and the electronics box, though care will have to be taken to avoid radiation and reception by the connecting wire that runs from one to the other. On the theoretical side, the magnitude of the residual perturbation when the sensor is in the plasma, and also the phenomena of radiation and reception by solid spherical electrodes of finite radius, need to be studied.

\section{SUMMARY AND CONCLUSIONS}

A new diagnostic method is proposed for determining, in a simple way, the electron density of a magnetoplasma from the amplitude of the mutual impedance of a mediumfrequency probe at the lower oblique resonance frequency.

The use of this method requires knowledge of the geometry of the probe, of its orientation with respect to the magnetic field, and of the electron temperature and gyrofrequency. These last two parameters can be deduced from the measured mutual impedance curve whenever an oscillatory structure, related to the temperature, and a main minimum, related to the gyrofrequency, occur simultaneously. When no such pecularities are in evidence, it is necessary to fit the theoretical curves to the experimental ones by trial and error. Alternatively, these two parameters may be obtained from other instruments, as was the case in the Porcupine F3 experiment.
In this experiment, the densities obtained from the data of the mf probe were too high due to the joint effects of stray capacitance in the sensor units, and of perturbation of the electrodes by the supporting structure; their influence was taken into account by using calibration measurements of the mutual impedance and self-impedance of the probe in a vacuum, obtained on the downleg of the flight.

In the future, at the price of some technical and theoretical developments, it should be feasible to dispense with calibration and to derive absolute values of the electron density from the measured values of the mutual impedance at the lower oblique resonance. If so, the $\mathrm{mf}$ probe would become an instrument to measure density with an accuracy comparable to that of the hf probe, and with at least three advantages: a narrower range of working frequencies, simpler and unambiguous data interpretation, and better sensitivity at low densities.

\section{ACKNOWLEDMENTS}

The authors thank all those who took part in the Porcupine program, in particular the principal investigator $G$. Haerendel and the co-investigators who provided them with advance copies of their results, especially J. M. Illiano and R. Pottelette.

This work was sponsored by the National Centre for Space Studies (C.N.E.S.).

\footnotetext{
'A. Gonfalone, J. Phys. (Paris) 33, 521 (1972).

${ }^{2}$ K. H. Burrell, Phys. Fuids 18, 1716 (1975).

${ }^{3}$ R. W. Boswell and J. Thiel, Phys. Fluids 24, 2120 (1981).

${ }^{4}$ A. Gonfalone, Radio Sci. 9, 1159 (1974).

${ }^{5}$ E. Michel, C. Béghin, A. Gonfalone, and I. F. Ivanov, Ann. Geophys. 31, $463(1975)$.

${ }^{6} \mathrm{~J}$. Thiel, P. Dorio, and R. Soubry, Comp. Phys. Comm. 23, 169 (1981).

'L. R. O. Storey and J. Thiel, Phys. Fluids 21, 2325 (1978).
} 